\title{
Teen Preferences for Clinic-Based Behavior Screens: Who, Where, When, and How? Jasik, $\mathrm{CB}^{1}$, Berna, $\mathrm{M}^{1}$, Martin, $\mathrm{M}^{2}$, Ozer, $\mathrm{EM}^{1,3}$
}

Affiliations: ${ }^{1}$ Division of Adolescent and Young Adult Medicine, Department of Pediatrics, University of California, San Francisco, ${ }^{2}$ The Ohio State University School of Medicine, Columbus, $\mathrm{OH},{ }^{3} \mathrm{UCSF}$ Office of Diversity and Outreach, University of California, San Francisco.

\begin{abstract}
Purpose: Previous research examining computer-based adolescent risk behavior screening was done prior to widespread adoption of smartphones and merits updating.

Methods: This is a cross-sectional survey among 115 adolescents seeking primary care age 1218. It is a diverse sample with $59 \%$ female, $51 \%$ white, $18 \%$ African-American, and $27 \%$ Latino. Respondents were asked level of comfort and honesty (1=strongly disagree, 5=strongly agree) when answering health behavior questions by paper, interview, or electronic device. Differences in the level of agreement tested using a Wilcoxon signed-rank test.

Results: Adolescents reported a higher level of comfort and honesty for screening conducted via electronic device vs. paper (90\% vs. 57\%, p<0.001; 89\% vs. 61\%, p<0.001). Sixty-two percent prefer waiting room electronic screening vs. at home $(4.7 \%)$ or by provider in the exam room (11.2\%).

Conclusions: Electronicsame-day risk behavior screening is the preferred method for adolescents and should be incorporated into preventive services.
\end{abstract}

Sources of Support: AHRQ R21 HS020997; Ozer - PI 


\section{Introduction}

Guidelines for adolescent preventive services recommend screening across a range of areas to reduce adolescent morbidity and mortality. Screening and counseling in key risk areas shows promise of improving behavioral outcomes in the areas of sexual behavior, diet and exercise, helmet use, substance use, and depression ${ }^{1}$; yet receipt of a preventive visit is as low as $38 \%$ and only one out of ten adolescents receive comprehensive preventive screening. ${ }^{2}$

Identified barriers to receipt of preventive screening include lengthy interviews, poor provider training, and low self-efficacy to deliver services to adolescents. ${ }^{3}$ Preventive screening increases rates of counseling for risk behaviorand are integral to effective interventions to influence adolescent behavior change. ${ }^{4}$ Existing technology interventions suggest that computer screening can adequately identify risk factors. ${ }^{5}$

Prior research, conducted before widespread adoption of the electronic health record (EHR) and smartphones, found that adolescent screening preferences are equivalent for paper or computer and merits updating. ${ }^{6}$ This study reports on adolescent preferences for preventive screening - specifically the method, location, and timing.

\section{Methods}

One hundred fifteen patients, age 12-18 years (mean 15 years), were recruited over 12 months in university-based primary care clinics as part of a study evaluating a tablet-based risk behavior screening module. The research assistant consented and screened patients registering for a well visit given the focus on preventive screening and counseling. Participants were 59\% female, 51\% white, 27\% Hispanic, 18\% African American, 17\% Asian, and 12\% other. 43\% of the group was age 12-14 and 57\% age 15-18. Baseline endorsement of risk behaviors ranged from $6 \%$ active smokers to $19 \%$ with a history of sexual activity. 
Participants completed a 21-item questionnairewhich assessedpreferences for screening and for this analysis, a subset of items was considered. Research procedures were approved by the UCSF Committee on Human Research (CHR). The primary outcome assessed was the preferred modality for psychosocial screening - electronic,provider interview, or paper.The survey incorporated a Likert scale (1=strongly disagree, $2=$ disagree, $3=$ neither agree or disagree, 4=agree, $5=$ strongly agree) based on agreement with how comfortable the patient was, and how honest they would be answering questions about health behaviors, when screened electronically, by provider interview, or with paper.

Other preferences measured were most preferred location (home, waiting room, exam room) and method for follow-up (e-mail, SMS, phone call, or none). Patients were also asked about use of and access to smartphones, computers, and tablets.Data were analyzed using the STATA software.Differences between screening preferences were tested using Wilcoxon signed rank test comparing percent who agree with a statement and paired t-tests of the mean comparing adolescents' honesty and comfort levels for answering questions about health behaviors by modality. Comparisons were made for provider interview and paper vs. electronic device.

\section{Results}

Table 1 shows adolescent preferences for preventive screening in terms of level of comfort and honesty in answering questions for paper and provider interview vs. electronic. The youth reported a strong preference for electronic screening with 90\% agreeing with comfort and $89 \%$ honesty vs. $57 \%$ and $61 \%$ for paper respectively. In addition, electronic screening preference was significantly different from provider interview, but the magnitude of the difference was less pronounced than for electronic vs. paper. Paper vs. provider interview was 
also tested and these significantly differed for the within group comparison and mean score testing.There was no difference in reported preference by demographic group (age or gender) or reported health risk behavior (alcohol, tobacco, drugs, or sexual activity).

Patients were also asked about their preference for location and timing of screening (Table 2). They reported the highest preference for electronic screening in the waiting room (61.6\%) vs. paper screen (8.4\%), in the exam room with the provider (11.2\%), or at home prior to the visit (4.7\%). Participants also reported a preference for post-visit follow-up via email (60.7\%) versus via text (16.8\%) or phone call (15\%). Thirty-two percent of respondents did not want the clinic to contact them at all post-visit. In terms of access to electronic devices at home, $82 \%$ of patients reported using a cell phone with internet access and $86 \%$ reported computers at home.

\section{Conclusions}

This study indicates that adolescents prefer electronicscreening and want the screening done in the waiting room vs. at home. And though they are open to post-visit follow-up, less invasive methods such as email are preferred. This is an interesting departure from prior research that suggested a preference for paper screening and only considered onescreening area. ${ }^{6}$ The results could be explained by the exponential increase in adolescent use of electronic devices for daily communication. Provider interview is also preferred vs. paper. This may speak to trust for the medical provider and research suggesting that adolescents want to discuss health behaviors with their provider. ${ }^{7}$ Our findings extend prior work by assessing adolescent preferences in the context of a clinically integrated electronic health screening tool vs. in the context of obtaining research data on risk behavior. ${ }^{8}$ 
As clinics move toward capitalizing on access to pre-visit questionnaires via electronic health records (EHR), it is important to understand when and how teens prefer to be screened. Research also indicates that teen are open to using EHR patient portals at home to communicate with their provider. ${ }^{9}$ Our results suggest within-clinic screening is preferred vs. at home, but they are open to e-mail communication post-visit.

Potential limitations of this study include the small sample size and location at an academic center. There may also be regional differences in level of tech savvy as this study was conducted in an urban population on the West Coast.

Research indicates that electronic screening takes less time than traditional methods. ${ }^{10}$ Given patient preference for this method, electronic screening is an efficient and comprehensive option for providers. The findings provide additional data supporting the transition from paper screening to electronic pre-visit methodsconducted in the waiting room,in combination with provider counseling during the visit. Future research should evaluate the impact of electronic screening applications on adolescent screening rates and provider acceptability. 


\section{References}

1. Ozer EM, Urquhart, JT, Brindis, CD, Park MJ, Irwin C. Young Adult Preventive Health Care Guidelines: There but Can’t Be Found. Arch Pediatr Adolesc Med. 2012;166(3):4-7.

2. Irwin CE, Adams SH, Park MJ, Newacheck PW. Preventive Care for Adolescents: Few Get Visits and Fewer Get Services. Pediatrics. 2009;123(4):e565-e572. doi:10.1542/peds.2008-2601.

3. Henry-Reid LM, O’Connor KG, Klein JD, Cooper E, Flynn P, Futterman DC. Current pediatrician practices in identifying high-risk behaviors of adolescents. Pediatrics. 2010;125(4):e741-e747. doi:10.1542/peds.2009-0271.

4. Ozer EM, Adams SH, Lustig JL, et al. Increasing the Screening and Counseling of Adolescents for Risky Health Behaviors: A Primary Care Intervention. Pediatrics. 2005;115(4):960-968. doi:10.1542/peds.2004-0520.

5. Harris SK, Csemy L, Sherritt L, et al. Computer-Facilitated Substance Use Screening and Brief Advice for Teens in Primary Care: An International Trial. Pediatrics. 2012;129(6):1072-1082. doi:10.1542/peds.2011-1624.

6. Knight JR, Harris SK, Sherritt L, Van Hook S, Lawrence N, Brooks T, Carey P, Kossack $\mathrm{R}$, Kulig J. Adolescents' preference for substance abuse screening in primary care practice. Substance Abuse. 2007: 28(4): 107-17.

7. Ford CA, Thomsen SL, Compton B. Adolescents' interpretations of conditional confidentiality assurances. J Adolesc Heal. 2001;29(3):156-159. doi:10.1016/S1054139X(01)00251-8.

8. Dolezal C, Marhefka SL, Santamaria EK, Leu C-S, Brackis-Cott E, Mellins CA. A comparison of audio computer-assisted self-interviews to face-to-face interviews of sexual behavior among perinatally HIV-exposed youth. Arch Sex Behav. 2012;41(2):401-410. doi:10.1007/s10508-011-9769-6.

9. Bergman DA, Brown NL, Wilson S. Teen use of a patient portal: a qualitative study of parent and teen attitudes. Perspectives in Health Information Management. 2008; 5:13.

10. Harris SK et al. Adolescent substance use screening in primary care: Validity of computer self-administered versus clinician-administered screening. Substance Abuse. 2016 JanMar;37(1):197-203. 
Table 1. Adolescent Preferences for Preventive Screening - Method (n=115)

\begin{tabular}{|c|c|c|c|c|c|c|}
\hline \multirow[b]{2}{*}{$\underline{\text { Method }}$} & \multicolumn{4}{|c|}{ Within-Group Comparisons } & \multicolumn{2}{|c|}{$\underline{\text { Mean Score }}^{\mathrm{b}}$} \\
\hline & $\underline{\text { Agree }}$ & $\underline{\text { Neutral }}$ & Disagree & p-value $^{\mathrm{a}}$ & Mean (SD) & p-value $^{c}$ \\
\hline $\begin{array}{l}\text { How comfortable I fee } \\
\text { about health behaviors }\end{array}$ & & & & & & \\
\hline Paper & $57.0 \%$ & $35.1 \%$ & $7.9 \%$ & $<0.001$ & $2.7(0.95)$ & $<0.001$ \\
\hline Provider Interview & $76.5 \%$ & $17.4 \%$ & $6.1 \%$ & 0.034 & $3.0(0.84)$ & 0.006 \\
\hline Electronic & $90.0 \%$ & $12.2 \%$ & $0.9 \%$ & - & $3.3(0.73)$ & - \\
\hline $\begin{array}{l}\text { How honest I feel ans } \\
\text { about health behaviors }\end{array}$ & & & & & & \\
\hline Paper & $60.9 \%$ & $33.9 \%$ & $5.2 \%$ & $<0.001$ & $2.8(0.89)$ & $<0.001$ \\
\hline Provider Interview & $73.9 \%$ & $20.0 \%$ & $6.1 \%$ & 0.006 & $3.0(0.87)$ & 0.002 \\
\hline Electronic & $88.7 \%$ & $10.4 \%$ & $0.9 \%$ & - & $3.3(0.68)$ & - \\
\hline
\end{tabular}

${ }^{a}$ Wilcoxon signed-rank test, comparing paper vs. electronic and interview vs. electronic.

${ }^{\mathrm{b}}$ Mean calculated based on 5 responses: $1=$ strongly disagree, $2=$ disagree, $3=$ neither, $4=$ =agree, $5=$ strongly agree.

${ }^{\mathrm{c}}$ Paired t-test of the means, comparing paper vs. electronic and interview vs. electronic. 
Table 2. Adolescent Preferences for Preventive Screening - Location and Timing ( $\mathrm{n}=115)$

Characteristic

Location

Before the visit - at home

Waiting Room - Electronic

Waiting Room - Paper

Exam Room - with provider

No preference

Post-Visit Follow-up ${ }^{\mathrm{a}}$

E-mail

Text

Phone Call

No follow-up
\%

$4.7 \%$

$61.6 \%$

$8.4 \%$

$11.2 \%$

$14.1 \%$

$60.7 \%$

$16.8 \%$

$15.0 \%$

$31.8 \%$

${ }^{\mathrm{a}}$ Some participants chose more than one answer. 